\section{OPEN ACCESS}

Edited by:

Fco. Pablo Holgado-Tello, National University of Distance

Education (UNED), Spain

Reviewed by:

Pedro Teques,

Polytechnic Institute of Maia, Portugal

Cristina Mazza,

Sapienza University of Rome, Italy

*Correspondence:

Blessings N. Kaunda-Khangamwa b.n.kaunda@gmail.com

Specialty section:

This article was submitted to

Quantitative Psychology

and Measurement,

a section of the journal

Frontiers in Psychology

Received: 15 February 2020

Accepted: 09 July 2020

Published: 31 August 2020

Citation:

Kaunda-Khangamwa BN,

Maposa I, Dambe R, Malisita K,

Mtagalume E, Chigaru L, Munthali A, Chipeta E, Phiri S and Manderson L

(2020) Validating a Child Youth

Resilience Measurement (CYRM-28)

for Adolescents Living With HIV

(ALHIV) in Urban Malawi.

Front. Psychol. 11:1896.

doi: 10.3389/fpsyg.2020.01896

\title{
Validating a Child Youth Resilience Measurement (CYRM-28) for Adolescents Living With HIV (ALHIV) in Urban Malawi
}

\begin{abstract}
Blessings N. Kaunda-Khangamwa ${ }^{1,2,3 *}$, Innocent Maposa', Rosalia Dambe ${ }^{4}$, Kennedy Malisita ${ }^{4}$, Emmanuel Mtagalume ${ }^{4}$, Lalio Chigaru ${ }^{4}$, Alister Munthali ${ }^{5}$, Effie Chipeta ${ }^{6}$, Sam Phiri ${ }^{2,7}$ and Lenore Manderson ${ }^{1,8,9}$
\end{abstract}

${ }^{1}$ School of Public Health, University of the Witwatersrand, Johannesburg, Johannesburg, South Africa, ${ }^{2}$ School of Public Health and Family Medicine, College of Medicine, University of Malawi, Blantyre, Malawi, ${ }^{3}$ Malaria Alert Centre, College of Medicine, University of Malawi, Blantyre, Malawi, ${ }^{4}$ Umodzi Family Centre, Blantyre, Malawi, ${ }^{5}$ Centre for Social Research, Chancellor College, University of Malawi, Zomba, Malawi, ${ }^{6}$ Centre for Reproductive Health, College of Medicine, University of Malawi, Blantyre, Malawi, ${ }^{7}$ Lighthouse Trust, Lilongwe, Malawi, ${ }^{8}$ School of Social Sciences, Monash University, Clayton, VIC, Australia, 9 Institute at Brown for Environment and Society, Brown University, Providence, RI, United States

Resilience as a strength-based notion, measured across cultures, age groups, and subpopulations, contributes to understanding health and well-being. Yet, there is limited evidence of how the construct performs in resource-limited countries. We explored the psychometric properties of the CYRM-28 and validated the scale with adolescents living with HIV (ALHIV), a key sub-population. The participants included members of an advisory panel and 406 ALHIV, aged 15-19 years, attending an antiretroviral therapy and teen-club clinic in Blantyre, Malawi. This study employed a mixed-method study using an exploratory sequential design. The advisory panel discussed the CYRM-28, and select ALHIV then translated it into Chichewa, pilot-tested it using cognitive interviews, and back-translated it for clarity and appropriateness. The resultant CYRM-28 was tested using a survey with purposefully selected ALHIV. The overall median score was 123, with an interquartile range of 110-130. Minimum and maximum scores were 53 and 140. Cronbach's alpha of 0.863 and Kaiser-Meyer-Olkin measure (0.866) confirmed internal consistency and the sample size adequacy, respectively. Bartlett's tests of sphericity $(\rho<0.001)$ informed factor analysis. Exploratory factor analysis determined possible dimensions of resilience and the sub-scales. The confirmatory factor analysis (CFA) confirmed the construct validity and supported a three-factor model consistent with the conceptualization of resilience as a multi-dimensional construct. Structural equation modeling was applied to perform CFA to measure model of resilience. Multiple fit indices showed a good fit for the model. The CYRM-28 has good internal consistency, 
test and re-test reliability, and moderate convergent validity which render it useful as a self-report resilience measure to inform and evaluate interventions for the health and well-being of adolescents in Malawi.

Keywords: Malawi, adolescents living with HIV, mixed methods, psychometric properties, resilience measures, teen-club clinic, validation

\section{INTRODUCTION}

Resilience describes doing well despite adversities. Resilience can be understood along a continuum where risks and protective factors interact, with individuals trying to reduce the effects of challenges or threats. Ungar defines resilience as "the capacity of individuals to navigate their way to the psychological, social, cultural, and physical resources that sustain their well-being, and their capacity individually and collectively to negotiate for these resources to be provided in culturally meaningful ways" (2008, 225). This definition encompasses multiple socio-ecological factors that influence positive outcomes over time. These are individual (cognitive skills, self-management), relational (peer, family, community support), and contextual (education, health, social services) (Obrist et al., 2010). The inclusion of socioecological factors in this study is critical for a nuanced understanding of resilience.

Globally, resilience researchers have used a variety of instruments to capture self-reported accounts of social well-being and health. Windle et al. (2011) and Henley (2010) identify that the most frequently used resilience tests and scales are COPE Scale, Rosenberg Self Esteem Scale, The Connor-Davidson Resilience Scale (Li et al., 2015), Child Youth Resilience Measure (CYRM) Resilience Scale (RS) (Sagone and De Caroli, 2014), and Antonovsky's Sense of Coherence Scale (SOC) (Windle et al., 2011). No scale or measurement is considered as superior, and there is a continuous debate on their feasibility, reliability, and validity in different settings (DeMichelis and Ferrari, 2016). However, the scales are biased toward personal constructs for adults and children in high-income countries, and there has been little work conducted in low- or middle-income settings (van Rensburg et al., 2017).

Studies on resilience with adolescents have predominantly focused on mental health, often with a single focus such as the role of peers, family, school, or community-based resources to improve both social and health outcomes (Moore et al., 2012). Ungar and Liebenberg (2011), however, discuss the significance of multiple formal and mandated interventions (mental health, education, child welfare, and the justice system) to foster resilience. Similarly, Betancourt et al. (2013) and van Breda and Theron, both working in sub-Sahara Africa, discuss the interplay between individuals and contexts, including access to resources, in understanding resilience (2013; 2018). The Resilience Research Centre ${ }^{1}$ recommends the use of CYRM-28 to capture contextual and cultural elements considered relevant to develop a valid measure of resilience (Ungar, 2016).

${ }^{1}$ www.resilienceresearch.org
There have been several attempts globally to validate the CYRM-28, including in Canada (Liebenberg et al., 2012), New Zealand (Sanders et al., 2017), Australia (Langham et al., 2018), and South Africa (van Rensburg et al., 2019). In the global north, there is variability in the factor structure correlations and relationships that measure resilience among youths (Pritzker and Minter, 2014). In South Africa, studies have been conducted on the analysis of resilience as a culturally and contextually entrenched construct among Sesotho-speaking youths (Van Breda and Theron, 2018), street youths (Malindi, 2014), and adolescents living with HIV (ALHIV) in KwaZulu Natal, using a factor structure analysis on youth experiencing sexual risks (Govender et al., 2017; van Rensburg et al., 2019). The aforementioned studies offer a rigorous cross-cultural adaptation process and psychometric analysis to reduce bias and allow for comparability of scores across cultures or populations (Jia and van de Vijver, 2015). However, methodological limitations within the CYRM include failure to load on one factor or sub-samples, and over-reliance on exploratory factor analysis (EFA) rather than confirmatory factor analysis (CFA) for the purposes of validation (Ungar and Liebenberg, 2011; Liebenberg et al., 2012). In addition to methodological limitations, findings on unique factors such as ubuntu and batho (community interdependence) as a source of resilience warrant further inquiry to understand concepts underlying resilience measurements among adolescents (Van Breda and Theron, 2018).

Ensuring that CYRM-28 measures are appropriate for ALHIV in Malawi is critical to identify factors and processes that characterize resilience in key sub-populations. Literature on resilience and HIV has particularly been informed by USAID programs under PEPFAR (United States President's Emergency Plan for AIDS Relief) (Fleischman and Peck, 2017). This includes the DREAMS (Determined, Resilient, Empowered, AIDS-free, Mentored, and Safe) initiative, which focused on reducing the incidence of HIV among adolescent girls and young women aged 15-24 (Brown et al., 2018; Saul et al., 2018). The DREAMS initiative centers on interventions that reduce the impact of structural drivers, such as HIV risk, poverty, gender inequality, sexual violence, and lack of education, to promote health and social outcomes among young women and male partners (Saul et al., 2018). Other studies include resourcing resilience through targeted social cash transfers to 13-22-year-olds, in Mchinji to improve school retention (Toska et al., 2016) and in Zomba district to reduce the risk of sexually transmitted infections, including HIV (Baird et al., 2012). These studies did not measure resilience per se, and so far there has been no assessment of the CYRM-28 factor structure among adolescents living with HIV in the country. An understanding of the resilience construct among this subpopulation will enhance the monitoring and 
development of relevant interventions to improve well-being and livelihoods of ALHIV.

\section{The CYRM-28 Factor Structure}

Despite the value of using CYRM-28, there is increasing evidence on the conflicting results from factor analysis, showing variability on the structure and scores, including the underlying construct (Liebenberg et al., 2012, 2017; van Rensburg et al., 2015). In their work with youth in Canada, Rensburg and colleagues used a 5-point Likert-type scale with three sub-scales (individual, relational, and context), and a 28 -item list with response options ranging from "not at all" to "a lot" (Liebenberg et al., 2012). On the other hand, a study with youth in New Zealand used four sub-scales and a 28-item list, with four measuring spirituality, seven items on individual resources, seven items on family resources, and ten items on social and cultural resources (Sanders et al., 2015). The South African structure of CYRM28 used with Sesotho-speaking youths combined social/cultural and communal/spiritual as one sub-scale, with less emphasis on individual sub-scales. Hence, resilience measures vary across context, sub-populations, and socio-cultural groups, warranting further investigation on validation processes, results, and interpretation (Windle et al., 2011; Jia and van de Vijver, 2015).

\section{MATERIALS AND METHODS}

\section{Study Design}

Data reported in this article derive from a non-interventional mixed-method study. The study employed an exploratory sequential design, with a two-phase method to inform and modify research tools. The study conformed to recommendations for using CYRM-28, which included employing a six-member advisory panel to inform research study processes, site-specific questions, and the adaptation of the scale for ALHIV in Malawi (Ungar, 2016). The original version of the CYRM was translated into Chichewa, the dominant language in the study setting, and this version was validated for survey use among ALHIV. Routine programmatic data from registers (attendance, gender, ages, in and out of care) from 2010 to 2019 informed the characteristics of the study participants and settings, and the discussion section of this article.

\section{Study Setting}

The study was conducted among ALHIV attending an Adolescent Teen Club at the Umodzi Family Centre (UFC) within Queen Elizabeth Central Hospital (QECH) in Blantyre, the major commercial city of Malawi. The QECH serves as a referral hospital for the south-west zone of the country. Adolescents attending the teen club, described in the next section, come from urban, peri-urban, and rural areas surrounding Blantyre. All adolescents testing HIV positive from these areas are counseled and offered enrollment in the UFC and other health centers and facilities surrounding QECH. Adolescents who need hospitalization are admitted to in-patient wards and are referred to UFC for HIV testing, confirmatory tests, and support. Before an adolescent is discharged, he or she receives another counseling session and is invited to join UFC or to attend an antiretroviral therapy (ART) clinic close to their home. As of June 30, 2019, the UFC ART clinic registry had a total of 14,160 people, of whom 3500 were adolescents aged 10-24.

\section{The Teen Club Program}

The UFC teen club program is a Lighthouse Trust, Youth Friendly Health Service initiative, managed by UFC and supported by bilateral partners. The club model was adapted from the United States Baylor College of Medicine International Pediatric AIDS Initiative (BIPAI) in partnership with the Malawi Ministry of Health (Baylor College of Medicine International Pediatric AIDS Initiative (BIPAI) 2012; MacKenzie et al., 2017). Adolescents aged 10-19 are eligible to participate in the club programs after disclosure which entails their acceptance of their HIV diagnosis, its implications, and their disclosure of this to significant others. Adolescents are prepared to transition out of the teen club program from around 20 years of age. Currently, teen club services adhere to a "test and treat" policy and provide differentiated service delivery in terms of service intensity, frequency, location, and health worker support. The emphasis is on a patientcentered care approach to HIV care (Prust et al., 2017; Chihana et al., 2018).

To strengthen the clinical, psychosocial, and resource supports, various activities are scheduled throughout the year. Lighthouse Trust manages these activities with support from the Ministry of Health, local non-governmental organizations, and PEPFAR under the Centers for Disease Control and Prevention (Phiri et al., 2017; Prust et al., 2017; Moody et al., 2018). The teen club is held on Saturdays and provides comprehensive programming for those aged 10-19 years under three pillars: (1) Clinical Care, including screening and management of opportunistic infections, ART monitoring including side effects and viral load, nutrition screening and treatment, TB screening and treatment, and referrals for specialized care; (2) Psychosocial Support: adherence counseling, peer support, mental health screening, coping with stigma and discrimination, livelihoods, and future planning; and (3) Sexual and Reproductive Health services: education and contraception administration (Kim et al., 2014; MacKenzie et al., 2017).

\section{Study Participants}

The study involved ALHIV, attending the UFC ART and teenclub clinic during the study period, with signed informed consent from parents/caregivers and assent from those aged 15-17 years, and informed consent from those aged 18-19 years. The study pool purposefully selected young people aged 15-19 because of the continued disproportionate effects on this group from HIV and the only age group to have experienced a rise in deaths caused by AIDS-related illness (UNAIDS, 2019). Participants included those attending (or not) the ART clinic and UFC teen club, in and out of school, pregnant or not, and included both those born with HIV and those horizontally infected. People excluded from the study included those who were critically ill, and those aged below 15 or above 19 years. 


\section{Sampling}

Sampling was based on similar studies conducted with adolescents attending Saturday teen-club clinics in Zomba and Lilongwe, in Malawi by Kim et al. (2015, 2017) and MacKenzie et al. (2017). Through the electronic medical records, we purposefully selected adolescents aged 15-19 between January 2018 and June 2019 attending the UFC ART clinic. Through the UFC database, a total of 1099 ALHIV were identified and invited to participate in the study. Follow-up revealed that 254 had transferred out, 3 had stopped attending the clinic, 379 had defaulted, and 57 were reported dead by October 2018. A total of 406 ALHIV who had been on ART for the past 90 days were eligible to participate (Figure 1).

\section{Recruitment Processes}

All adolescents attending the UFC ART and teen-club clinics were invited to take part in the study when they presented at the clinic reception. The support staff working and adolescents' mentors at the ART clinic gave out letters of invitation written in vernacular, Chichewa. They explained briefly the study objectives to all adolescents aged 15-19, over an 8-month period (November 2018-June 2019). Adolescents were given a chance to be recruited as they would normally visit the clinic for routine follow-up and attend the teen club every month or two. There were two different letters of invitations for adolescents aged 1517 to attend, with their parents signing the consent form while they signed an assent form; those aged 18 and 19 provided a written consent form.

\section{Instruments, Measures, and Procedures Site-Specific Questions}

Consistent with the CYRM manual regarding site-specific questions to capture context, relevant questions were conceptualized during the pilot survey and shared with the panel of six advisors as part of triangulation (Ungar, 2016). There were 11 questions, including statements on life experiences and adolescents' knowledge of service use, for example: "Being an adolescent is challenging," "I can handle difficulties in my life," "I have knowledge of sexual and reproductive health," and "the teen-club clinic provides support." The 11 site-specific items were measured on a 5-point Likert scale, ranging from 1 (not at all) to 5 (a lot) (Appendix 1).

\section{CYRM-28}

The adapted version of the CYRM-28 (Liebenberg et al., 2012) was administered to measure ALHIV resilience. Resilience is a latent variable that comprises 28 items with eight components (individual: personal and social skills, peer support; caregiver

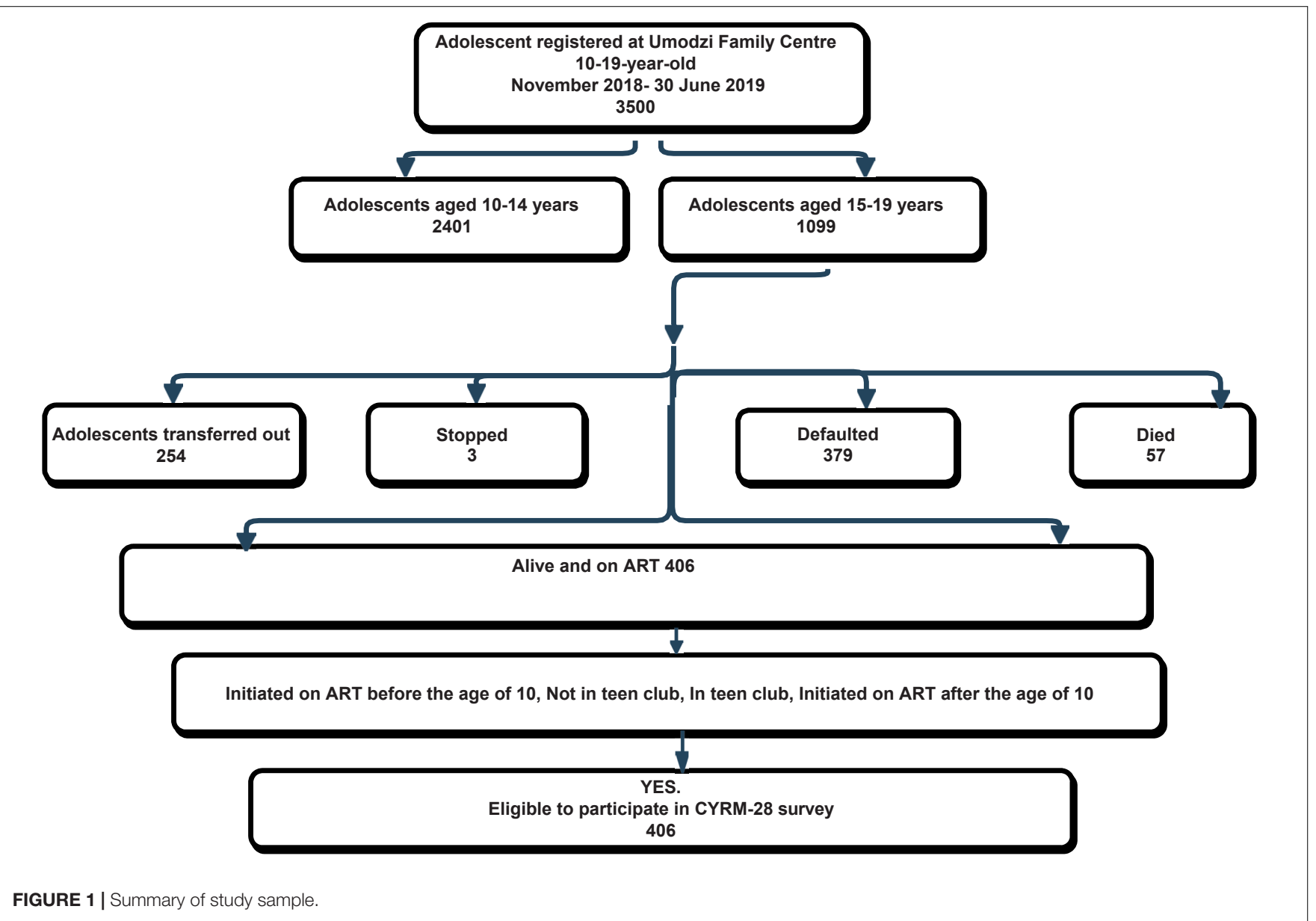


physical and psychological and context: spiritual, education, and cultural), which provide insights to three subscales (Ungar, 2016). The individual sub-scale has 11 items that are divided under (1) personal skill with (5 items), peer support (2 items), and social skill (4 items); (2) the relational sub-scale considers physical and psychological caregiving with 2 and 5 items, respectively; and (3) the context sub-scale focuses on spirituality ( 3 items), education (2 items), and culture (5 items) (van Rensburg et al., 2017). The following are some examples of statements under individual personal skills - "I am able to solve problems without hurting myself or others"; caregiving - "My caregiver stands by me during difficult times"; context - "I enjoy my community's traditions" (Ungar, 2016).

In this article, we concentrate on adaptation processes and changes to the wording of the CYRM-28 items. These modifications are consistent with the manual to ensure context relevance and familiarity with competing measures (van Rensburg et al., 2015, 2017). The items are measures on a 5point Likert scale, ranging from 1 (not at all) to 5 (a lot) with higher scores $(\leq 140)$ representing increased resilience (Ungar and Liebenberg, 2011). A summary of these 28 items within their sub-scales is set out in Appendix 2.

\section{Data Collection}

Data collection occurred in two phases. The first phase was a pilot and pre-test study, using cognitive interviews with 42 participants to determine if ALHIV understood the questions and underlying concepts in the CYRM-28. The interviews were held in Zomba (70 km from Blantyre) at Tisungane teen club, Zomba Central Hospital, as a rapport-building activity; we targeted cognitive processes to ensure that the questions worked as intended (Zukerberg and Hess, 1996; Ungar, 2008).

Previous studies on cognitive interviewing have used "think aloud" and verbal probing techniques with a group of 5-10 subjects to discuss concepts and question-wording formats (Blair and Brick, 2010). In this study, we held the first three rounds of cognitive interviews among young people aged 15-16, 1718 , and 19 years. We employed verbal probing techniques with six adolescents in each groups. The remaining four rounds were spread over 3 weeks to follow up questions, probe on meanings of resilience, explore the reasons for varied responses, and gather insight on clarity of the questions to improve the English and Chichewa interview guides (Heather et al., 2012).

To minimize bias in both the pilot and post-pilot studies, the first author trained the same four research assistants on the purpose of the study, objectives, and processes to administer the survey. The research assistants were part of the mentorship program under UFC team and underwent three rounds of completing the CYRM form with feedback sessions to ensure standardized administration of the questionnaire (Jia and van de Vijver, 2015). In addition, weekly and monthly meetings were held with the data collection team to ensure consistency in the language used and instructions to complete the survey.

The pilot established a draft of context-specific questions, which incorporated the CYRM-28. This was presented and discussed with the advisory panel to ensure contextual relevance. Slight changes were made to the wording as all questions were translated into the vernacular to ensure that adolescents were able to understand the questions. A single survey questionnaire with the modified CYRM-28 was then drafted (Appendix 2).

The second phase of data collection took place with individuals and in groups in Blantyre from November 2018 to June 2019. The final questionnaire was administered to 406 adolescents from UFC ART and teen-club clinics in Blantyre. The questionnaire as described previously was structured and included both open- and close-ended and Likert-scale questions (Windle et al., 2011; van Rensburg et al., 2015).

The adolescents completed the paper-based questionnaire in a classroom set-up in the lecture hall at the College of Medicine through 20 group sittings (9-20 adolescents each), with some individuals participating on other days to participate and enable us to reach our required sample size. Each question was read aloud to participants, with questions clarified if needed, by the first author. The respondents were given a minute to write down their responses to each statement. The research assistants helped those adolescents who independently filled in the questionnaire to ensure that they had understood all questions and completed the questionnaire. The four adolescents who had writing and hearing problems, respectively, were helped by the research assistants present at each group sitting. The likelihood of experiencing bias in the results are minimal because the ALHIV were required to tick most of the responses in the questionnaire.

\section{Statistical Data Analysis}

Quantitative data were captured from the completed paper questionnaires into a REDCap electronic data entry form. REDCap is a secure, web-based application designed to support research data capture, and provides space for validated data entry and automated export procedures to STATA version 14.2 in readiness for analyses (Harris et al., 2009). Descriptive analysis was performed on demographic and psychometric measures to summarize characteristics of participants. Minimum, maximum, and ranges were reported for all relevant variables. Medians and interquartile range were reported for skewed continuous variables, and means and standard deviations for continuous and normally distributed variables. Frequencies and percentages were used to describe the categorical variables.

Reliability measures like Cronbach's alpha were used to assess the internal consistency of the resilience measurement scale. A Cronbach's alpha $\geq 0.70$ indicates satisfactory reliability (Tavakol and Dennick, 2011). Kaiser-Meyer-Olkin (KMO) measure and Bartlett's tests of sphericity were used to assess the adequacy of our sample size and inter-correlations between items to warrant factor analysis (Govender et al., 2017). A KMO closer to 1.0 confirms the feasibility for factor analysis (Acock, 2013; Pritzker and Minter, 2014).

The reasons EFA was used in this study include (1) to ascertain the factor structure of the resilience measurement scale, inclusive of all sub-scales (individual, caregiver, and context) to ensure that all items in the scale measured resilience as an underlying construct (Langham et al., 2018) and (2) because our questionnaire items were not normally distributed but were skewed. The CFA using structural equation 
modeling (SEM) in Stata 14.2 assessed the relationships and inter-relationships between different latent factors of the CYRM-28 of observed and measured behaviors (Acock, 2013). In addition, the CFA determines how the resilience measurement scale conformed to the original conceptual framework (Goretzko et al., 2019). Steps are set out in Figure 2.

The maximum-likelihood estimation method was used to estimate the CFA model. Multiple indices were used to assess the model fit: root mean square error of approximation (RMSEA), Akaike's Information Criterion (AIC), Bayesian Information Criterion (BIC), Tucker-Lewis Index (TLI), Comparative Fit Index (CFI), and Standardized Root Mean Squared Residual (SRMR) (Govender et al., 2017). Values greater than 0.95 indicate excellent fit to the data whereas values greater than 0.90 indicate acceptable fit to the data (de Carvalho and Chima, 2014). In addition, the average variance extracted (AVE) was estimated to evaluate convergent validity with values greater than 0.50 indicating convergent validity as part of exhibiting evidence in full (Sawatzky et al., 2017).

\section{RESULTS}

A total of 406 ALHIV aged 15-19 years participated, with almost equal numbers of males and females. Their mean age was 16.74, SD 1.46. There was heterogeneity between ethnic and religious groups, although the dominant ethnic groups were Lhlomwe,
Chewa, and Ngoni (64\% of total participants). Only 3.0\% ALHIV did not know their tribe. Religious affiliation, school attendance, access to meals per day, and sleeping spaces varied (Table 1).

Overall, the median score for resilience was 123, with an interquartile range of 110-130. The minimum and maximum scores were 53 and 140, with most scores concentrated on the higher side. Five adolescents attained the maximum score of 140 and only one recorded 53. No adolescent scored the lowest possible score of 28. Bartlett's test of sphericity, 2766.820, $p<0.001$, showed that our variables had high correlation, which warranted factor analysis. The KMO measure of sampling adequacy was 0.866, confirming that our sample was good enough to continue with EFA (Acock, 2013). The alpha, which is a measure of reliability and showed the internal consistency of the measured dimension, recorded 0.862 for the overall resilience construct whereas the subscales showed individual component, $\alpha=0.733$; relational component, $\alpha=0.702$; and contextual component, $\alpha=0.667$. Results in Table 2 show internal reliability for measures of reliability and construct reliability. However, alpha can be high even with items that are minimally related to one another. Therefore, we ran a factor analysis to show correlations between dimensions that represent a shared meaning among the 28-item CYRM measure (Liebenberg et al., 2012; Mampane, 2014).

The EFA through factor analysis showed three factors with eigenvalues above 1 . The eigenvalues were obtained for each factor to identify a measure of variance. The first component

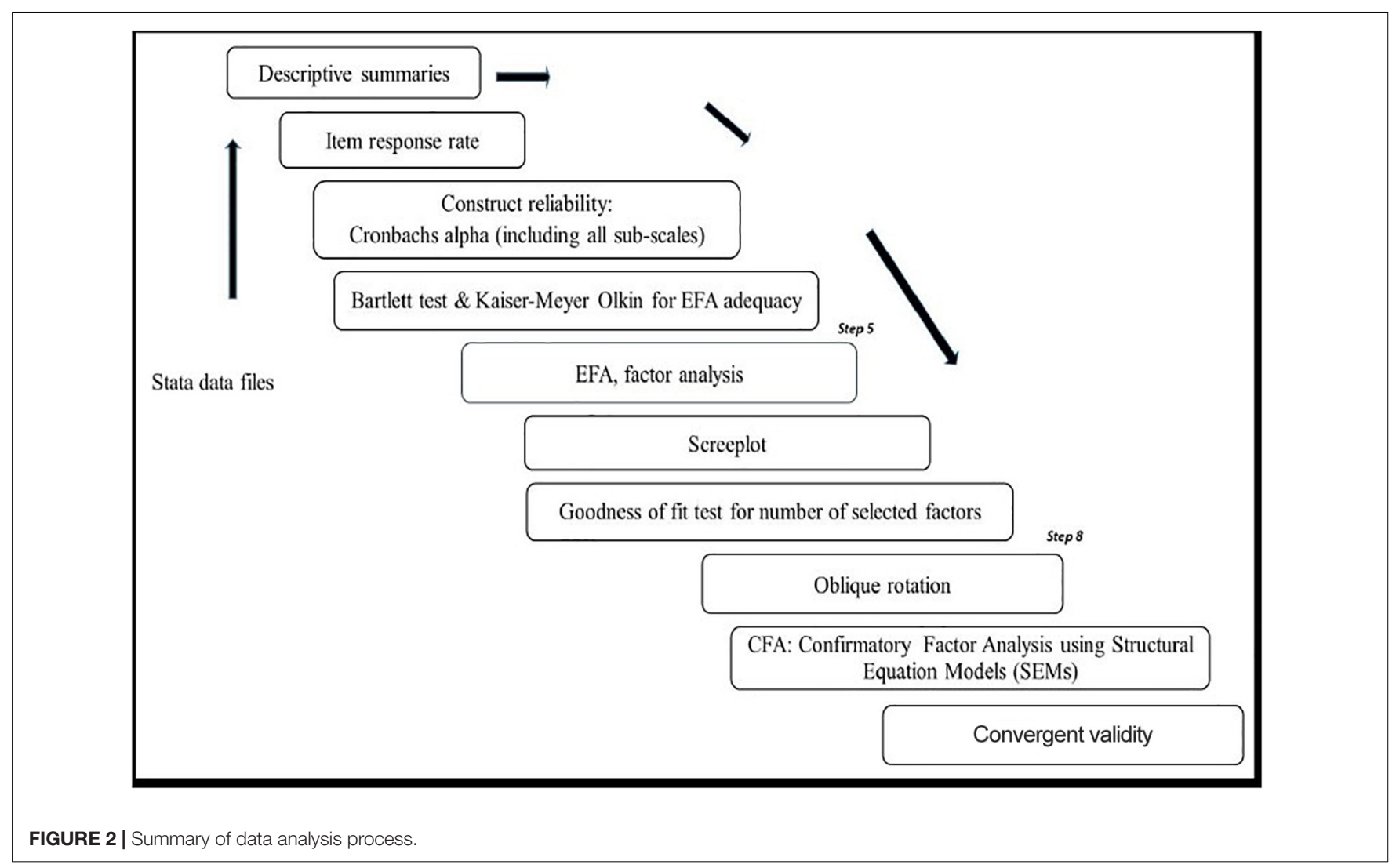


TABLE 1 | Summary of socio-demographic characteristics of participants.

\begin{tabular}{|c|c|c|c|}
\hline Variable & Overall N (\%) & Male $n(\%)$ & Female $n(\%)$ \\
\hline & $406(100)$ & $204(50.2)$ & $202(49.8)$ \\
\hline Mean (SD) & $1.5(0.50)$ & $1.48(0.50)$ & $1.55(0.40)$ \\
\hline \multicolumn{4}{|l|}{ Age } \\
\hline $15-16$ & 196 (48.28) & $91(44.61)$ & 105 (51.98) \\
\hline 17-19 & 210(51.72) & 97 (48.02) & 113(55.39) \\
\hline \multicolumn{4}{|l|}{ Education } \\
\hline None & $38(9.45)$ & 19(50) & 19(50) \\
\hline Primary & 141(35.07) & $82(58.16)$ & $59(41.84)$ \\
\hline Secondary & $223(55.47)$ & 102(45.74) & $121(54.26)$ \\
\hline \multicolumn{4}{|l|}{ Ethnic Group } \\
\hline Chewa & $80(19.70)$ & $42(52.50)$ & $38(47.50)$ \\
\hline Tonga & $18(4.43)$ & 10(55.56) & $8(44.44)$ \\
\hline Sena & $26(6.40)$ & $15(42.32)$ & 11(57.68) \\
\hline Lhlomwe & $101(24.88)$ & $45(44.55)$ & $56(55.45)$ \\
\hline Ngoni & 79 (19.46) & $43(54.43)$ & $36(45.57)$ \\
\hline Tumbuka & 40(9.85) & $20(50)$ & $20(50)$ \\
\hline Yao & $34(8.37)$ & $17(50)$ & $17(50)$ \\
\hline Mang'anja & $12(2.96)$ & 4(33.33) & $8(66.67)$ \\
\hline Don't Know & $12(2.96)$ & $6(50)$ & $6(50)$ \\
\hline Other & $4(0.99)$ & $2(50)$ & $2(50)$ \\
\hline \multicolumn{4}{|l|}{ Religion } \\
\hline Protestants & $205(50.49)$ & 108(52.68) & $97(47.32)$ \\
\hline Catholics & $83(20.44)$ & $38(45.78)$ & $45(54.22)$ \\
\hline Moslems & $28(6.90)$ & $17(60.71)$ & 11(39.29) \\
\hline Pentecostals & $86(21.18)$ & $39(45.35)$ & $47(54.65)$ \\
\hline Other & 4 (0.99) & $2(50)$ & $2(50)$ \\
\hline \multicolumn{4}{|l|}{ Ever had a baby } \\
\hline Yes & $12(2.9)$ & $3(1.5)$ & $9(4.5)$ \\
\hline No & $394(97)$ & 201(98) & 193(95) \\
\hline \multicolumn{4}{|l|}{ Death in the family } \\
\hline Yes & 253(63.3) & $129(64.2)$ & $124(62.3)$ \\
\hline No & $147(36.57)$ & $72(35.8)$ & $75(37.7)$ \\
\hline \multicolumn{4}{|l|}{ Person who died } \\
\hline Mother & $78(31.2)$ & $35(27.6)$ & $43(34.9)$ \\
\hline Father & 98(39.2) & $52(40.9)$ & $46(37.4)$ \\
\hline Brother & 24(9.6) & 15(11.8) & $9(7.3)$ \\
\hline Sister & $19(7.6)$ & $9(7.0)$ & 10(8.3) \\
\hline Grandparent & $4(2)$ & $3(2.3)$ & $1(1)$ \\
\hline Aunt & $18(7.2)$ & $11(8.7)$ & $7(5.7)$ \\
\hline \multicolumn{4}{|l|}{ Ever moved houses } \\
\hline Yes & $207(50.9)$ & $104(50.2)$ & $103(49.8)$ \\
\hline No & 199(49.0) & $100(50.3)$ & $99(49.8)$ \\
\hline \multicolumn{4}{|c|}{ Frequency of changing homes } \\
\hline Once & $63(30.7)$ & $31(49.2)$ & $32(50.7)$ \\
\hline Twice & 53(25.9) & $25(47.2)$ & $28(52.8)$ \\
\hline Three or more times & $67(32.7)$ & $37(55.2)$ & $30(44.8)$ \\
\hline I do not know & 19(9.3) & $8(42.1)$ & 11(57.9) \\
\hline Other & $3(1.5)$ & $2(66.6)$ & 1(33.3) \\
\hline \multicolumn{4}{|l|}{ Sleeping spaces } \\
\hline Alone & $167(41.2)$ & $91(54.5)$ & $76(45.5)$ \\
\hline With 2 or three people & $120(29.6)$ & $61(50.8)$ & $59(49.2)$ \\
\hline More than 4 people & $84(20.7)$ & $36(42.9)$ & $48(57.4)$ \\
\hline I do not know & $24(5.9)$ & $12(50)$ & $12(50)$ \\
\hline
\end{tabular}

TABLE 1 | Continued

\begin{tabular}{lccc}
\hline Variable & Overall N (\%) & Male $\mathbf{n}(\%)$ & Female n (\%) \\
\hline Other & $10(2.5)$ & $4(40)$ & $6(60)$ \\
Number of meals per day & & & \\
One meal & $11(2.7)$ & $6(54.6)$ & $5(45.5)$ \\
Two meals & $47(11.6)$ & $29(61.7)$ & $18(38.3)$ \\
Three meals or more & $322(79.5)$ & $155(48.1)$ & $167(51.2)$ \\
I do not know & $19(4.7)$ & $11(57.8)$ & $8(42.1)$ \\
Other & $6(1.5)$ & $5(50)$ & $3(50)$ \\
\hline
\end{tabular}

showed an eigenvalue of 5.63 , out of 28 items, and explained $63 \%$ of the variance. The second and third factors recorded 1.41 and 1.1 eigenvalues explaining $79 \%$ variance of the second factor, whereas $92 \%$ explained the total variability of the three factors. In addition, we used the scree plot to confirm the three points of inflection for the final analysis (Ledesma et al., 2015).

Consistent with previous studies on the conceptualization of resilience as a dynamic process and a multi-dimensional construct over time (Liebenberg et al., 2012; Govender et al., 2017; van Rensburg et al., 2019), we computed the factor analysis with oblique rotation (direct oblimin) to accentuate and show the rotated correlations on the three components (individual, relational, and contextual) that make up the resilience measure. Two out of 28 items (items 1 and 15) did not load: "I have people I look up to" and "I know where to go in my community to get help." Four items of the CYRM-28 had factor loadings $<0.350$ (items $11,13,17$, and 28) showing inconsistencies on the role of individual and contextual components in informing resilience across cultures.

We again performed factor analysis on the three-factor model with eigenvalues greater than 1 . Factor loadings in the three-factor solution showed that 15 items loaded on factor 1 , whereas 10 items and 3 items loaded on factor 2 and factor 3, respectively. One item - "I feel supported by friends" loaded on both factors 1 and 3, and one item - "I am treated fairly in my community" - loaded on both factors 2 and 3. Table 3 shows the three-factor solution under the three subscales after rotation. Ten items and seven items loaded on individual and relational components. Nine items out of 10 loaded on the contextual component. Twenty-six items in the three-factor model had good loadings of $>0.3$, a minimum criterion for threshold loadings (Acock, 2013; Ledesma et al., 2015). Based on theoretical models of resilience, therefore, we adopted a three-factor structure to better explain resilience among ALHIV (Liebenberg et al., 2012; Gale et al., 2013; Pritzker and Minter, 2014).

The internal reliability of sub-scales was re-assessed using alpha and sample adequacy by KMO, which retained similar results, confirming items for the CFA. This allowed the analysis of the sub-scales to show similar results as in the initial EFA model. Hence, the decision to retain all 28 items for a resilience measure was informed by reliability tests, EFA, and a socio-ecological framework that reflects on individual, relational, and contextual interactions and supports 
TABLE 2 | Alpha reliability.

\begin{tabular}{|c|c|c|c|c|c|}
\hline Item & Sign & it-cor & is-cor & ii-cov & alpha \\
\hline 1. I have people I look up to in my life & + & 0.514 & 0.454 & 0.29838 & 0.887 \\
\hline 2. I cooperate with people around me & + & 0.478 & 0.417 & 0.30079 & 0.888 \\
\hline 3. Getting an education is important to me & + & 0.511 & 0.467 & 0.30401 & 0.887 \\
\hline 4. I know how to behave in different social situations & + & 0.482 & 0.429 & 0.30285 & 0.888 \\
\hline 5. My parent(s)/caregiver(s) watch me closely & + & 0.616 & 0.581 & 0.30087 & 0.886 \\
\hline 6. My parents/caregivers know a lot about me & + & 0.535 & 0.489 & 0.30164 & 0.887 \\
\hline 7. If I am hungry, there is enough to eat at home & + & 0.488 & 0.429 & 0.30069 & 0.888 \\
\hline 8. I try to finish what I start & + & 0.554 & 0.508 & 0.30004 & 0.886 \\
\hline 9. Spiritual beliefs are a source of strength for me. & + & 0.567 & 0.519 & 0.29868 & 0.886 \\
\hline 10. I am proud of my tribe's background & + & 0.550 & 0.508 & 0.30227 & 0.888 \\
\hline 11. People think I am funny to be with & + & 0.520 & 0.474 & 0.30274 & 0.887 \\
\hline 12. I talk to my family/caregiver(s) about how I feel & + & 0.528 & 0.472 & 0.29878 & 0.887 \\
\hline 13. I can solve problems without harming myself or others by using drugs or violence & + & 0.400 & 0.320 & 0.3025 & 0.891 \\
\hline 14. I feel supported by my friends & + & 0.477 & 0.408 & 0.29854 & 0.889 \\
\hline 15. I know where to go in my community to get help & + & 0.446 & 0.367 & 0.2989 & 0.890 \\
\hline 16. I feel I belong at my school & + & 0.535 & 0.473 & 0.29556 & 0.887 \\
\hline 17. My family stands by me during difficult times. & + & 0.519 & 0.463 & 0.29898 & 0.887 \\
\hline 18. My friends stand by me during difficult times. & + & 0.472 & 0.402 & 0.29886 & 0.889 \\
\hline 19. I am treated fairly in my community & + & 0.531 & 0.466 & 0.2951 & 0.887 \\
\hline 20. I have opportunities to show others that I am becoming an adult and can act responsibly & + & 0.539 & 0.485 & 0.29826 & 0.887 \\
\hline 21. I am aware of my own strengths & + & 0.538 & 0.494 & 0.30225 & 0.888 \\
\hline 22. I participate in organized religious activities & + & 0.586 & 0.539 & 0.29698 & 0.886 \\
\hline 23. I think it is important to serve my community & + & 0.530 & 0.581 & 0.30097 & 0.887 \\
\hline 24. I feel safe when I am with my family/caregivers. & + & 0.531 & 0.487 & 0.30259 & 0.887 \\
\hline 25. I have opportunities to develop skills useful in life, like job skills and skills to care for others). & + & 0.499 & 0.441 & 0.30013 & 0.888 \\
\hline 26. I enjoy my family's/caregiver's cultural and family traditions & + & 0.511 & 0.460 & 0.30142 & 0.887 \\
\hline 27. I enjoy my community's tradition & + & 0.514 & 0.452 & 0.29237 & 0.888 \\
\hline 28. I am proud to be Malawian & + & 0.403 & 0.369 & 0.31258 & 0.889 \\
\hline Test scale & & & & 0.30045 & $\begin{array}{l}0.891^{\star} \\
\text { mean }\end{array}$ \\
\hline
\end{tabular}

to enable resilience over time (van Rensburg et al., 2015; Ungar, 2016).

\section{Confirmatory Factor Analysis}

The confirmatory factor analysis confirmed the three-factor structure (individual, relational, and contextual) of CYRM-28 with clustered items (see Figure 3).

Statistical methods normally use one statistical test to determine the significance of analysis as well as model fit (Ungar, 2016; Le et al., 2017). Our study employed multiple indices rather than a single index to confirm a model fit to ensure our study results will be comparable at local and international arenas (see Appendix 3) for factor-level analysis and comparable results for our model. The overall fit for the source of resilience model after covariance is illustrated as $\chi^{2}(p<0.001)$, $\mathrm{df}=1$, RMSEA $=0.06$, SRMR $=0.03$, TLI $=961, \mathrm{CFI}=982, p=0.002$, $\mathrm{AIC}=14,287.937$, and $\mathrm{BIC}=14,412.133$. There is a need for flexibility when using multiple indices to apply the goodness-offit guidelines to inform decision-making in practice (Govender et al., 2017; van Rensburg et al., 2019). Hence, the $p$ value, TLI, CFI, SRMR, and RMSEA showed a relatively adequate fit for our model. The interpretability of the fit indices is significant and provided space to understand correlations across and within the sub-scales, including the overall resilience construct.

The factor loadings in the model were positive, and the standardized loadings were high $(>0.60)$ except for individual: peer support (0.45) and contextual: education (0.59). This suggests that all three latent variables were significant and positively correlated in the sample (Liebenberg et al., 2012; Acock, 2013). The CI for the correlation between the corresponding subscales of context and caregiving shows (0.82) and caregiving and individual components recording $(0.62,0.85)$ and $(0.72,0.91)$ for context and individual sub-scales. The strong correlations reflect the presence of resilience as an underlying construct. All items, factors, and sub-scales in this Malawian model were statistically significant $(p \leq 0.001)$. Hence, our model confirms that the sub-scales did measure resilience. Composite reliability $(\mathrm{CR})$, and convergent and discriminant validity were performed after fitting the model as part of validating resilience construct in full (Ungar and Liebenberg, 2011). Two dimensions exceeded 0.50 ( 0.616 individual and 0.50 caregiving), whereas the context recorded 0.38 and had problems with convergent validity. All three constructs had discriminant validity problems (this was expected since all constructs are more related than different (Table 4). 
TABLE 3 | Pattern matrix of the three-factor solution for the CYRM-28.

\begin{tabular}{|c|c|c|c|}
\hline \multicolumn{4}{|c|}{ Components } \\
\hline & Individual & Relational & Context \\
\hline \multicolumn{4}{|l|}{ 1. I have people I look up to in my life } \\
\hline \multicolumn{4}{|l|}{$\begin{array}{l}\text { 15. I know where to go in my } \\
\text { community to get help }\end{array}$} \\
\hline 2. I cooperate with people around me & 0.423 & & \\
\hline 8. I try to finish what I start & 0.576 & & \\
\hline $\begin{array}{l}\text { 4. I know how to behave in different } \\
\text { social situations }\end{array}$ & 0.553 & & \\
\hline 11. People think I am funny to be with & 0.343 & & \\
\hline $\begin{array}{l}\text { 13. I can solve problems without } \\
\text { harming myself or others by using } \\
\text { drugs or violence }\end{array}$ & 0.341 & & \\
\hline 14. I feel supported by my friends & 0.498 & & \\
\hline $\begin{array}{l}\text { 18. My friends stand by me during } \\
\text { difficult times. }\end{array}$ & 0.440 & & \\
\hline $\begin{array}{l}\text { 20. I have opportunities to show others } \\
\text { that I am becoming an adult and can } \\
\text { act responsibly }\end{array}$ & 0.447 & & \\
\hline 21. I am aware of my own strength & 0.485 & & \\
\hline $\begin{array}{l}\text { 24. I feel safe when I am with my } \\
\text { family/caregiver }\end{array}$ & 0.474 & & \\
\hline $\begin{array}{l}\text { 25. I have opportunities to develop } \\
\text { skills useful in life, like job skills and } \\
\text { skills to care for others). }\end{array}$ & 0.568 & & \\
\hline $\begin{array}{l}\text { 5. My parent(s)/caregiver(s) watch me } \\
\text { closely }\end{array}$ & & 0.581 & \\
\hline $\begin{array}{l}\text { 6. My parents/caregivers know a lot } \\
\text { about me }\end{array}$ & & 0.499 & \\
\hline $\begin{array}{l}\text { 7. If I am hungry, there is enough to eat } \\
\text { at home }\end{array}$ & & 0.547 & \\
\hline $\begin{array}{l}\text { 12. I talk to my family/caregiver(s) about } \\
\text { how I feel }\end{array}$ & & 0.417 & \\
\hline $\begin{array}{l}\text { 17. My family stands by me during } \\
\text { difficult times. }\end{array}$ & & 0.313 & \\
\hline $\begin{array}{l}\text { 26. I enjoy my family's/caregiver's } \\
\text { cultural and family traditions }\end{array}$ & & 0.590 & \\
\hline $\begin{array}{l}\text { 3. Getting an education is important to } \\
\text { me }\end{array}$ & & & 0.649 \\
\hline $\begin{array}{l}\text { 9. Spiritual beliefs are a source of } \\
\text { strength for me. }\end{array}$ & & & 0.522 \\
\hline $\begin{array}{l}\text { 10. I am proud of my tribe's } \\
\text { background }\end{array}$ & & & 0.593 \\
\hline 16. I feel I belong at my school & & & 0.336 \\
\hline 19. I am treated fairly in my community & & & 0.446 \\
\hline $\begin{array}{l}22 . \text { I participate in organized religious } \\
\text { activities }\end{array}$ & & & 0.637 \\
\hline $\begin{array}{l}\text { 23. I think it is important to serve my } \\
\text { community }\end{array}$ & & & 0.511 \\
\hline 27. I enjoy my community's tradition & & & 0.580 \\
\hline 28. I am proud to be Malawian & & & 0.327 \\
\hline
\end{tabular}

\section{DISCUSSION}

The purpose of this study was to explore the factor structure of the CYRM-28 in a sub-population of ALHIV aged 15-19 and receiving teen-club clinic support in Malawi. This was part of a broader objective to validate the CYRM- 28 by assessing the adaptation processes which included slight changes in the wording and translation to vernacular Chichewa. Our study results showed varied perceptions of components and scales contributing to their resilience. In particular, ALHIV perceptions differed on the individual peer support and education supports. This is consistent with previous studies that argue that across sub-populations, there are common resilience elements, but the way they are grouped is different (Langham et al., 2018; Van Breda, 2018). Overall, our study demonstrates construct validity (dependent on measurement scores, (1) content; (2) item responses; and (3) relations to other dimensions, e.g., convergent, discriminant, concurrent (Ungar and Liebenberg, 2011; Pritzker and Minter, 2014), informs gaps in HIV and resilience research (van Rensburg et al., 2015; de Araújo et al., 2017), and highlights the need to be mindful of the role of peers, community, and educational support in the lives of ALHIV. This suggests the value of further qualitative research.

Items 1 and 15, in relation to individual personal and social skills, failed to load in the CYRM-28. This failure of factor loadings is not new; studies using the similar English-language version of CYRM-28 in Canada and South Africa failed on items 1, 5, and 28 (Liebenberg et al., 2012; Govender et al., 2017). The items that failed to load - "I have people I look up to" and "I know where to go in my community to get help" - may reflect the exceptional nature of HIV/AIDS (Moyer and Hardon, 2014). Fear, social exclusion, self-stigma, and social stigma and discrimination continue to affect the everyday lives of people in Malawi, and this ultimately influences people's interactions with services. However, in line with our theoretical notions on the socio-ecological framework, we retained all statements on CYRM 28-item to measure and understand resilience at multiple levels (Van Breda and Theron, 2018).

In ALHIV research in Malawi, there are mixed outcomes of the role of peers and education in improving well-being and resilience (Amzel et al., 2013; Toska et al., 2016). For instance, Baird et al. (2012) and Toska et al., 2016 have observed that conditional cash transfers linked to school enrollment encourage young people staying in school longer, and in association, this lowered teen pregnancies. However, in our study, some adolescents might have underreported their access to educational support as part of the need for incentives for their education. Low factor loadings were also recorded in previous studies in South Africa, Canada, and Australia on items 1 and 28, which provides more evidence on some of the problematic items that make up the CYRM-28 (Ungar, 2016; Govender et al., 2017; Langham et al., 2018). Our study highlights caregiving support and spiritual and cultural supports as key to informing resilience among ALHIV attending ART clinics in Malawi. Focusing on physical and psychological caregiving, adolescents' spiritual and cultural supports through religion and rites of passage have been identified as key protective factors for ALHIV (Van Breda and Theron, 2018; OECD Development Centre, 2018).

The results from modeling and resilience demonstrated problems of convergent validity, evidenced by low to moderate correlations between dimensions. The challenge with convergent and discriminant validity may be a result of the fact that the 


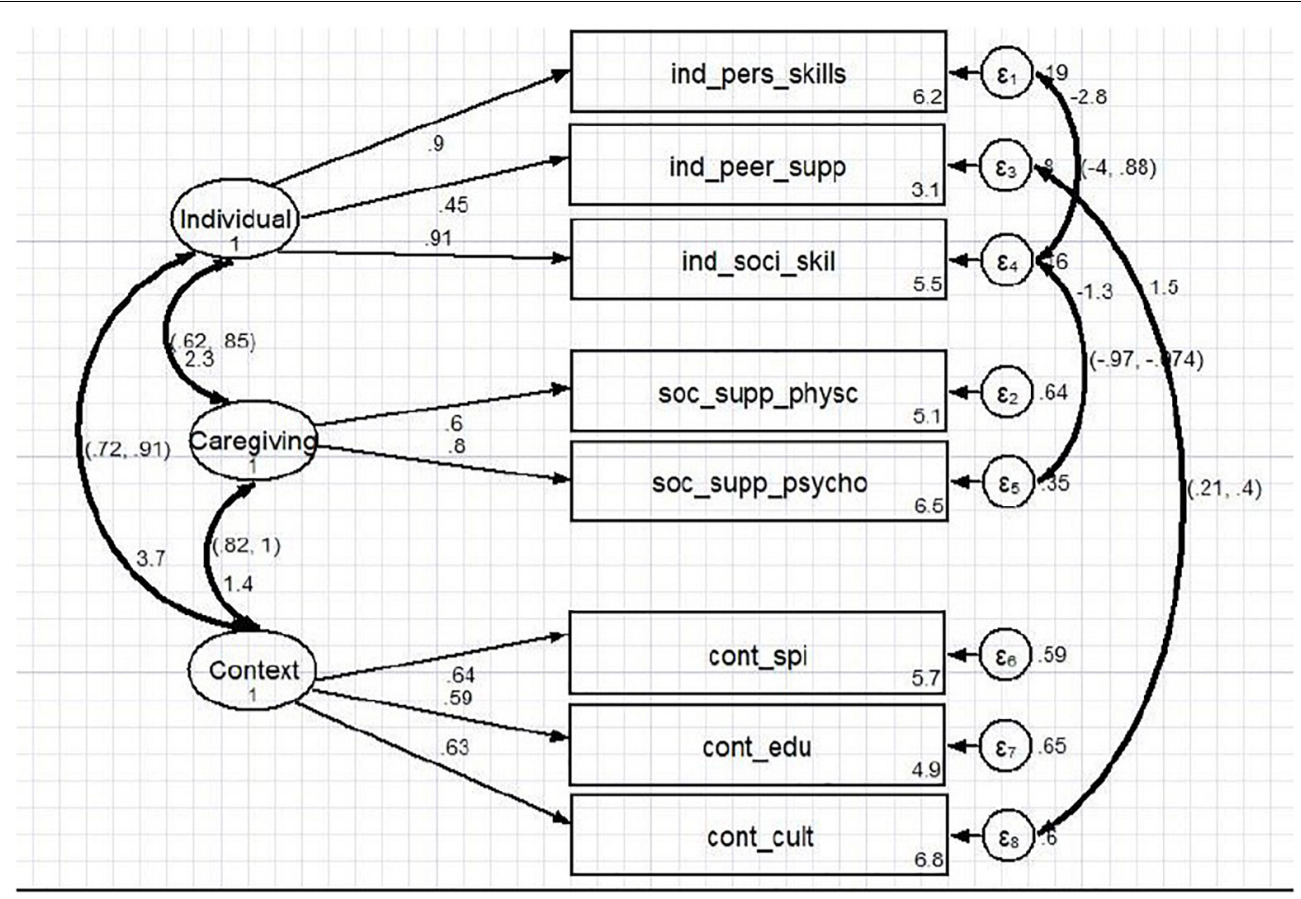

FIGURE 3 | Confirmatory factor analytic model.

TABLE 4 | Factor loadings, composite reliability, and average variance extracted.

\begin{tabular}{lccc}
\hline Construct/sub-scale & $\begin{array}{c}\text { Factor } \\
\text { loadings }\end{array}$ & $\begin{array}{c}\text { Composite } \\
\text { reliability (CR) }\end{array}$ & $\begin{array}{c}\text { Average variance } \\
\text { extracted (AVE) }\end{array}$ \\
\hline $\begin{array}{l}\text { Individual } \\
\text { - Personal skills }\end{array}$ & 0.90 & 0.817 & 0.616 \\
- Peer support & 0.45 & & \\
- Social skills & 0.91 & & \\
Caregiving & & 0.665 & 0.502 \\
- Physical & 0.60 & & \\
- Psychological & 0.80 & & \\
Context & & 0.655 & \\
- Spiritual & 0.64 & & \\
- Education & 0.59 & & \\
- Cultural & 0.63 & & \\
\hline
\end{tabular}

constructs are composed by few indicators and were being assessed in the same model (Byrne, 2012). However, using SEM showed that individual, relational, cultural, and spiritual contexts are closely correlated. The individual personal and social skills work hand in hand with psychological caregiving whereas cultural and individual peer support factors interact for a better fit. The possible explanation for this is that in some cultures, the status of "being an adolescent" may be connected to less prestige and less respect than that of adults. Previous studies on resilience among ALHIV in South Africa have shown the role of community interdependence as illustrated by ubuntu/batho concepts as key factors underlying resilience (Theron and Theron, 2010; Van Breda, 2018). In our study, ALHIV did not identify as a person in and of the community, but rather, they identified as individuals, with less emphasis on peer connectedness and community membership as sources of resilience owing to the stigma and discrimination of living with HIV. In addition, in relation to discrimination, most societies are adult centered, meaning that the needs and preferences of adults are given priority compared with those ones of adolescents whose specific interests are not taken into account. Further qualitative research should elaborate on how ALHIV express and interpret their resilience.

\section{IMPLICATIONS}

Current validation studies on resilience note the need to use the CYRM-28 to identify resilience-enabling sources and resources across cultures and sub-population (Sanders et al., 2015; Masten, 2016; van Rensburg et al., 2019). This study was conducted among adolescents accessing ART and psychosocial support at a teen-club clinic. This study foreshadows the resilience profiles of ALHIV contextually, and so responds to the need to be sensitive on how ALHIV interact with individual, relational, and sociocultural resources and dynamics as key sources for resilience.

The provision of services under the model of a one-stop shop or the differentiated service delivery approach to meet ALHIV complex needs is key to improving resilience-related outcomes (MacKenzie et al., 2017). Adapting and validating the CYRM28 for ALHIV in Malawi allowed for the description of various factors or resources that they recognize and interact with for better program planning and implementation. Future research might address the usefulness of the CYRM-28 in measuring intervention outcomes. 


\section{Strength and Limitations}

This study provides preliminary evidence on the use of CYRM28 in Malawi. By adopting the strength-based approach in this study, we looked at ALHIV interacting in meaning and decision-making processes regarding individual, relational, and resourceful supports in their lives. However, this assessment was a once-off process in a single context, among adolescents attending a teen-club clinic. Caution should be applied when extending the results to the general adolescent population. Although we captured resilience among ALHIV with a heterogeneous cultural background, for comprehensive evidence on validation, there is a need to consider adolescents across sub-populations, including those living without HIV, to enrich our findings and to help generalize research and intervention implications.

\section{CONCLUSION}

Our study contributed to technical and statistical evidence around using CYRM-28 to measure factors and processes underlying resilience among ALHIV in a low-income country. The findings supported the three-factor structure comprising 28 items representing individual, relational, and community/spiritual dimensions. The CYRM is a reliable measure of adolescent resilience, although additional research may be required to validate the instruments among alternative populations and adolescent sub-groups in Malawi.

\section{DATA AVAILABILITY STATEMENT}

All datasets generated for this study are included in the article/Supplementary Material.

\section{ETHICS STATEMENT}

The studies involving human participants were reviewed and approved by the Human Research Ethics Committee (HREC) of University of the Witwatersrand, Johannesburg, South Africa and the College of Medicine Research and Ethics Committee (COMREC), Blantyre, Malawi. The ethics clearance numbers for HREC and COMREC are M180465 and P.04/18/2389, respectively, are M180465 and P.04/18/2389,

\section{REFERENCES}

Acock, A. (2013). Discovering Structural Equation Modeling Using Stata. Texas: STATA Press.

Amzel, A., Toska, E., Lovich, R., Widyono, M., Patel, T., Foti, C., et al. (2013). Promoting a combination approach to paediatric HIV psychosocial support. AIDS 27, S147-S157. doi: 10.1097/QAD.0000000000000098.Promoting

Baird, S. J., Garfein, R. S., McIntosh, C. T., and Özler, B. (2012). Effect of a cash transfer programme for schooling on prevalence of HIV and herpes simplex type 2 in Malawi: a cluster randomised trial. Lancet 379, 1320-1329. doi: 10. 1016/S0140-6736(11)61709-1

Baylor College of Medicine International Pediatric Aids Initiative (BIPAI) (2012). Baylor College of Medicine International Pediatric. (AIDS)Initiative-Malawi respectively. Written informed consent to participate in this study was provided by the participants' legal guardian/next of kin. Written informed consent was obtained from the individual(s), and minor(s)' legal guardian/next of kin, for the publication of any potentially identifiable images or data included in this manuscript.

\section{AUTHOR CONTRIBUTIONS}

BK-K conceived, designed, and managed the study protocol. $\mathrm{BK}-\mathrm{K}, \mathrm{RD}, \mathrm{KM}, \mathrm{EM}$, and LC undertook the field collections and contributed to the write-up on study setting and teen-club programmes. BK-K, IM, RD, KM, AM, EC, SP, and LM focused on both qualitative and quantitative analyses and interpretation. BK-K, IM, and LM conceived, designed, managed, analyzed data, and wrote the manuscript. All authors read, edited, and approved the final version of the manuscript.

\section{FUNDING}

This research was supported by the Consortium for Advanced Research Training in Africa (CARTA). CARTA is jointly led by the African Population and Health Research Center and the University of the Witwatersrand and funded by the Carnegie Corporation of New York (Grant No-B 8606.R02), Sida (Grant No:54100113), the DELTAS Africa Initiative (Grant No: 107768/Z/15/Z), and Deutscher Akademischer Austauschdienst (DAAD). The DELTAS Africa Initiative is an independent funding scheme of the African Academy of Sciences (AAS)'s Alliance for Accelerating Excellence in Science in Africa (AESA) and supported by the New Partnership for Africa's Development Planning and Coordinating Agency (NEPAD Agency) with funding from the Wellcome Trust (United Kingdom) and the United Kingdom government. The statements made and views expressed are solely the responsibility of the Fellow.

\section{SUPPLEMENTARY MATERIAL}

The Supplementary Material for this article can be found online at: https://www.frontiersin.org/articles/10.3389/fpsyg. 2020.01896/full\#supplementary-material

Teen Club Curriculum Part 1: Content A Resource for Groups working with Adolescents Living with HIV. Texas: BIPAI.

Betancourt, T., Meyers-Ohki, S., Charrow, A., and Hansen, N. (2013). Mental health and resilience in HIV/AIDS affected children: a review of the literature and recommendations for future research. J. Child Psychol. Psychiatry 54, 423-444. doi: 10.1111/j.1469-7610.2012.02613.x. Mental

Blair, J., and Brick, P. D. (2010). "Methods for the analysis of cognitive interviews," in Proceedings of the Section on Survey Research Methods - JSM2010 (Alexandria, VA: American Statistical Association), 3739-3748.

Brown, K., Williams, D. B., Kinchen, S., Saito, S., Radin, E., and Patel, H. (2018). Status of HIV epidemic control among adolescent girls and young women aged 15 - 24 Years - Seven African Countries, 2015 - 
2017. Morb. Mortal. Weekly Rep. 67, 29-32. doi: $10.15585 / \mathrm{mmwr}$. mm6701a6

Byrne, B. M. (2012). Structural Equation Modeling With M plus. Basic Concepts, Applications, and Programming. Abingdon: Routledge.

Chihana, M., Huerga, H., Cutsem, G. Van, Ellman, T., Wanjala, S., Masiku, C., et al. (2018). Impact of " test and treat " recommendations on eligibility for antiretroviral treatment: cross sectional population survey data from three high HIV prevalence countries. PLoS One 13:e0207656. doi: 10.1371/journal.pone. 0207656

de Araújo, L. F., Teva, I., Quero, J. H., Reyes, A. O., and de la Paz Bermúdez, M. (2017). Analysis of resilience and sexual behavior in persons with HIV infection. Psicologia 30:21. doi: 10.1186/s41155-017-0076-6

de Carvalho, J., and Chima, F. O. (2014). Applications of structural equation modeling in social sciences research. Am. Int. J. Contemp. Res. 4, 6-11.

DeMichelis, C., and Ferrari, M. (eds) (2016). "Child and adolescent resilience within medical contexts," in Integrating Research and Practice. Child and Adolescent Resilience Within Medical Contexts: Integrating Research and Practice, (Berlin: Springer), doi: 10.1007/978-3-319-32223-0

Fleischman, J., and Peck, K. (2017). Addressing HIV in Adolescent Girls and Young Women in Malawi: The DREAMS Partnership. Avaliable at: https: //csis-prod.s3.amazonaws.com/s3fs-public/publication/170717_Fleischman_ AddressingHIVMalawi_Web.pdf?qr_KhNHDjqyqf8cqJLqeeFIUIP_88j40.

Gale, N. K., Heath, G., Cameron, E., Rashid, S., and Redwood, S. (2013). Using the framework method for the analysis of qualitative data in multi-disciplinary health research. BMC Med. Res. Methodol. 13:117. doi: 10.1186/1471-2288-13117

Goretzko, D., Thien, T., Pham, H., and Bühner, M. (2019). Exploratory factor analysis: current use, methodological developments and recommendations for good practice. Curr. Psychol. 2, 1-12. doi: 10.1007/s12144-019-00 $300-2$

Govender, K., Cowden, R. G., Asante, K. O., George, G., and Reardon, C. (2017). Validation of the child and youth resilience measure among South African adolescents. PLoS One 12:e0185815. doi: 10.1371/journal.pone.0185815

Harris, P. A., Taylor, R., Thielke, R., Payne, J., Gonzalez, N., and Conde, J. G. (2009). Research electronic data capture (REDCap)- A metadata-driven methodology and workflow process for providing translational research informatics support. J. Biomed. Inform. 42, 377-381. doi: 10.1016/j.jbi.2008.08.010

Heather, H., Lambert, A. D., Kinzie, J., and Gieser, J. (2012). Cognitive Interviews to Improve Survey Instruments. Annual Forum of the Association for Institutional Research. Avaliable at http://cpr.indiana.edu/uploads/ AIR2012CognitiveInterviews.pdf. (accessed January 3, 2020).

Henley, R. (2010). Resilience enhancing psychosocial programmes for youth in different cultural contexts: evaluation and research. Prog. Dev. Stud. 10, 295307. doi: 10.1177/146499340901000403

Jia, H., and van de Vijver, F. (2015). "The value of keeping an open eye for methodological issues in research on resilience and culture," in Youth Resilience and Culture: Commonalities and Complexities, eds L. Theron and L. Liebenberg (New York, NY: Springer), 189-201. doi: 10.1007/978-94-017-9415-2_14

Kim, M. H., Mazenga, A. C., Yu, X., Devandra, A., Nguyen, C., Ahmed, S., et al. (2015). Factors associated with depression among adolescents living with HIV in Malawi. BMC Psychiatry 15:264. doi: 10.1186/s12888-015-0649-9

Kim, M. H., Mazenga, A. C., Yu, X., Devandra, A., Nguyen, C., Ahmed, S., et al. (2017). High self-reported non-adherence to antiretroviral therapy amongst adolescents living with HIV in Malawi: barriers and associated factors. J. Int. AIDS Soc. 20, 1-12. doi: 10.7448/IAS.20.1.21437

Kim, S.-H., Gerver, S. M., Fidler, S., and Ward, H. (2014). Adherence to antiretroviral therapy in adolescents living with HIV: systematic review and meta-analysis. AIDS 28, 1945-1956. doi: 10.1097/QAD.000000000 0000316

Langham, E., McCalman, J., Redman-MacLaren, M., Hunter, E., Wenitong, M., Britton, A., et al. (2018). Validation and factor analysis of the child and youth resilience measure for indigenous australian boarding school students. Front. Public Health 6:299. doi: 10.3389/fpubh.2018.00299

Le, M. T. H., Tran, T. D., Holton, S., Nguyen, H. T., Wolfe, R., and Fisher, J. (2017). Reliability, convergent validity and factor structure of the DASS-21 in a sample of Vietnamese adolescents. PLoS One 12:e0180557. doi: 10.1371/journal.pone. 0180557
Ledesma, R. D., Valero-Mora, P., and Macbeth, G. (2015). The scree test and the number of factors: a dynamic graphics approach. Spanish J. Psychol. 18:E11. doi: $10.1017 /$ sjp. 2015.13

Li, X., Chi, P., Sherr, L., Cluver, L., and Stanton, B. (2015). Psychological resilience among children affected by parental HIV/AIDS: a conceptual framework. Health Psychol. Behavi. Med. 3, 217-235. doi: 10.1080/21642850.2015.1068698

Liebenberg, L., Joubert, N., and Foucault, M.-L. (2017). Understanding Core Resilience Elements and Indicators a Comprehensive Review of the Literature. Avaliable at http://indaliebenberg.com/wp-content/uploads/2017/11/PHACResilience-Report-Final-Version-November-2017.pdf (accessed February 3, 2020).

Liebenberg, L., Ungar, M., and Van De Vijver, F. (2012). Validation of the child and youth resilience measure-28 (CYRM-28) among Canadian youth. SAGE J. 22, 219-226. doi: 10.1177/1049731511428619

MacKenzie, R. K., van Lettow, M., Gondwe, C., Nyirongo, J., Singano, V., Banda, V., et al. (2017). Greater retention in care among adolescents on antiretroviral treatment accessing "Teen Club" an adolescent-centred differentiated care model compared with standard of care: a nested case-control study at a tertiary referral hospital in Malawi. J. Int. AIDS Soc. 20:e25028. doi: 10.1002/jia2. 25028

Malindi, M. J. (2014). Swimming upstream in the midst of adversity: exploring resilience-enablers among street children. J/ Soc. Sci. 39, 265-274. doi: 10.1080/ 09718923.2014.11893289

Mampane, M. R. (2014). Factors contributing to the resilience of middleadolescents in a South African township: Insights from a resilience questionnaire. South Afr. J. Educ. 34, 1-11. doi: 10.15700/201412052114

Masten, A. (2016). Resilience in developing systems: the promise of integrated approaches. Eur/ J. Dev. Psychol. 13, 297-312. doi: 10.1080/17405629.2016. 1147344

Moody, L., Nicholls, B., Shamji, H., Bridge, E., Dhanju, S., and Singh, S. (2018). The person-centred care guideline: from principle to practice. J. Patient Exp. 5, 282-288. doi: 10.1177/2374373518765792

Moore, M. L., Westley, F. R., and Nicholls, A. (2012). The social finance and social innovation nexus 1. J. Soc. Entrepreneurship 3, 115-132. doi: 10.1080/19420676. 2012.725824

Moyer, E., and Hardon, A. (2014). A disease unlike any other? why hiv remains exceptional in the age of treatment. Med. Anthropol. 33, 263-269. doi: 10.1080/ 01459740.2014 .890618

Obrist, B., Pfeiffer, C., and Henley, R. (2010). Multi-layered social resilience: a new approach in mitigation research. Prog. Dev. Stud. 10, 283-293. doi: 10.1177/ 146499340901000402

OECD Development Centre (2018). Youth Well-Being Policy Review of Malawi”, EU-OECD Youth Inclusion Project. Paris: OECD.

Phiri, S., Neuhann, F., Glaser, N., Gass, T., Chaweza, T., and Tweya, H. (2017). The path from a volunteer initiative to an established institution: evaluating 15 years of the development and contribution of the Lighthouse trust to the Malawian HIV response. BMC Health Serv. Res. 17:548. doi: 10.1186/s12913-017-2466-y

Pritzker, S., and Minter, A. (2014). Measuring adolescent resilience: an examination of the cross-ethnic validity of the RS-14. Child. Youth Serv. Rev. 44, 328-333. doi: 10.1016/j.childyouth.2014.06.022

Prust, M. L., Banda, C. K., Nyirenda, R., Chimbwandira, F., Kalua, T., Jahn, A., et al. (2017). Multi-month prescriptions, fast-track refills, and community ART groups: results from a process evaluation in Malawi on using differentiated models of care to achieve national HIV treatment goals. J. Int. AIDS Soc. 20(Suppl. 4), 41-50. doi: 10.7448/IAS.20.5.21650

Sagone, E., and De Caroli, M. E. (2014). Relationships between psychological wellbeing and resilience in middle and late adolescents. Proc. Soc. Behav. Sci. 141, 881-887. doi: 10.1016/j.sbspro.2014.05.154

Sanders, J., Munford, R., and Liebenberg, L. (2017). Positive youth development practices and better outcomes for high risk youth. Child Abuse Neglect 69, 201-212. doi: 10.1016/j.chiabu.2017.04.029

Sanders, J., Munford, R., Thimasarn-Anwar, T., Liebenberg, L., and Ungar, M. (2015). The role of positive youth development practices in building resilience and enhancing wellbeing for at-risk youth. Child Abuse Neglect 42, 40-53. doi: 10.1016/j.chiabu.2015.02.006

Saul, J., Bachman, G., Allen, S., Toiv, N. F., and Beamon, C. C. T. (2018). The DREAMS core package of interventions: a comprehensive approach 
to preventing HIV among adolescent girls and young women. PLoS One 13:e0208167. doi: 10.1371/journal.pone.0208167

Sawatzky, R., Sajobi, T. T., Brahmbhatt, R., Chan, E. K. H., Lix, L. M., and Zumbo, B. D. (2017). "Social indicators research series 69. longitudinal change in response processes: a response shift perspective," in Understanding and Investigating Response Processes in Validation Research, Vol. 69, eds B. D. Zumbo and A. M. Hubley (Berlin: Springer), 1-378. doi: 10.1007/978-3-319-5 6129-5

Tavakol, M., and Dennick, R. (2011). Making sense of Cronbach's alpha. Int. J. Med. Educ. 5, 53-55. doi: 10.5116/ijme.4dfb.8dfd

Theron, L. C., and Theron, A. M. C. (2010). A critical review of studies of South African youth resilience, 1990-2008. South Afr. J. Sci. 106, 1-8. doi: $10.4102 /$ sajs.v106i7/8.252

Toska, E., Gittings, L., Hodes, R., Cluver, L. D., Govender, K., Chademana, K. E., et al. (2016). Resourcing resilience: social protection for HIV prevention amongst children and adolescents in Eastern and Southern Africa. Afr. J. AIDS Res. 15, 123-140. doi: 10.2989/16085906.2016.1194299

UNAIDS (2019). UNAIDS DATA . Avaliable at: https://www.unaids.org/sites/ default/files/media_asset/2019-UNAIDS-data_en.pdf (accessed January 23, 2020).

Ungar, M. (2008). Resilience across cultures. Br. J. Soc. Work 38, 218-235. doi: $10.1093 /$ bjsw/bcl343

Ungar, M. (2016). The Child and Youth Resilience Measure (CYRM) Child Version. Users Manual. Halifax: Resilience Research Centre.

Ungar, M., and Liebenberg, L. (2011). Assessing resilience across cultures using mixed methods: construction of the child and youth resilience measure. J. Mixed Methods Res. 5, 126-149. doi: 10.1177/15586898114 00607

Van Breda, A. D. (2018). A critical review of resilience theory and its relevance for social work. Soc. Work 54, 1-20. doi: 10.15270/54$1-611$
Van Breda, A. D., and Theron, L. C. (2018). A critical review of South African child and youth resilience studies, 2009-2017. Child. Youth Serv. Rev. 91, 1-28. doi: 10.1016/j.childyouth.2018.06.022

van Rensburg, A., Theron, L., and Rothmann, S. (2015). A review of quantitative studies of South African youth resilience: some gaps. South Afr. J. Sci. 111, 1-9. doi: 10.17159/sajs.2015/20140164

van Rensburg, A., Theron, L. C., and Ungar, M. (2019). Using the CYRM-28 with south african young people: a factor structure analysis. Res. Soc. Work Pract. 29, 93-102. doi: 10.1177/1049731517710326

van Rensburg, A. C., Theron, L. C., and Ungar, M. (2017). Using the CYRM-28 with south african young people: a factor structure analysis. Res. Soc. Work Pract. 29, 93-102. doi: 10.1177/1049731517710326

Windle, G., Bennett, K. M., and Noyes, J. (2011). A methodological review of resilience measurement scales. Health Q. Life Outcomes 9:8. doi: 10.1186/14777525-9-8

Zukerberg, A., and Hess, J. (1996). "Uncovering adolescent perceptions: experiences with conducting cognitive interviews with adolescents," in Proceedings of the Survey Research Methods Section of the American Statistical Association (Alexandria, VA: American Statistical Association), 950-955.

Conflict of Interest: The authors declare that the research was conducted in the absence of any commercial or financial relationships that could be construed as a potential conflict of interest.

Copyright (C) 2020 Kaunda-Khangamwa, Maposa, Dambe, Malisita, Mtagalume, Chigaru, Munthali, Chipeta, Phiri and Manderson. This is an open-access article distributed under the terms of the Creative Commons Attribution License (CC BY). The use, distribution or reproduction in other forums is permitted, provided the original author(s) and the copyright owner(s) are credited and that the original publication in this journal is cited, in accordance with accepted academic practice. No use, distribution or reproduction is permitted which does not comply with these terms. 